\title{
Innovation et société : pour élargir l'analyse des effets territoriaux de l'innovation ${ }^{1}$
}

\section{Innovation and Society: Broadening the Analysis of the Territorial Effects of the Innovation}

\author{
Jean-Marc Fontan ${ }^{\mathrm{a}}$, Juan-Luis Klein ${ }^{\mathrm{b} *}$, \\ Diane-Gabrielle Tremblay ${ }^{\mathrm{c}}$ \\ ${ }^{a}$ CRISES et Département de sociologie, Université du Québec à Montréal, CP888, \\ Centre-Ville, Montréal (Québec), Canada, H3C $3 P 8$. \\ ${ }^{b}$ CRISES et Département de géographie, Université du Québec à Montréal, CP888, \\ Centre-Ville, Montréal (Québec), Canada, H3C3P8. \\ ${ }^{c}$ CRISES et Département d'économie, TELUQ, Université du Québec, 4750, Henri-Julien, \\ Montréal (Québec), Canada, H2T3E4
}

\section{Résumé}

Traditionnellement, l'innovation a été associée aux technologies et a été abordée dans un cadre individuel ou entrepreneurial. Ce texte soutient que l'innovation s'inscrit dans un processus plus large, porteur d'un ensemble de dimensions. Le processus d'innovation est certes technique, mais il est aussi social. Étudier une innovation, c'est mettre en lumière les ressources sociales, techniques,

Adresse e-mail : Jean-Marc Fontan : Fontan.jean-marc@uqam.ca,

* Auteur correspondant.

Adresse e-mail : Juan-Luis Klein : Klein.juan-luis@uqam.ca,

1 Les auteurs tiennent à remercier le Conseil des recherches en sciences humaines (CRSH) du Canada pour leur appui financier. Ils remercient aussi le collègue Norbert Alter pour ses commentaires sur une version préliminaire de ce texte.

Adresse e-mail : Diane-Gabrielle Tremblay : dgtremblay@teluq.uquebec.ca, 
économiques, politiques et culturelles qui sont mobilisées par des acteurs individuels et collectifs. Cet ensemble d'éléments trouve corps et sens du fait qu'il prend place à une période et en un lieu précis. Dès lors, tant la dimension territoriale que la dimension temporelle sont importantes et donc à prendre en considération, car elles sous-tendent ce que les auteurs appellent « innovation socioterritoriale ».

() 2004 Lavoisier, Paris. Tous droits réservés.

\begin{abstract}
Traditionally, the concept of innovation has been associated to the world of technologies and has been analyzed in an individual or entrepreneurial context. This paper proposes that innovation is embedded in a much larger and multidimensional process. Certainly, the innovation is a technologic one, but it is also a social one. Analyzing an innovation implies considering social, technical, economical and cultural resources mobilized by individual and collective actors. All those elements of the social innovation process are temporally and territorially located, which represent two dimensions of what the authors call "socio-territorial innovation".
\end{abstract}

(c) 2004 Lavoisier, Paris. Tous droits réservés.

Mots clés : Innovation ; Innovation sociale ; Acteurs sociaux ; Changement social ; Territoire.

Keywords: Innovation; Social innovation; Social actors; Social change; Territory.

Le concept d'innovation est communément restreint au domaine des technologies, voire de la technique. Jusqu'aux années 1990, rares étaient ceux qui parlaient d'innovation sociale, sauf, dans certains cas, pour mentionner l'incidence probable du social sur l'émergence de l'innovation technique. L'analyse s'arrêtait là. Cet article vise à élargir l'analyse de l'innovation en proposant une réflexion sur sa dimension sociale, notion en usage depuis peu dans la mouvance de la «nouvelle sociologie économique » (Lévesque et al., 2001) et de la « géographie socio-économique » (Benko et Lipietz, 2000). Le point de vue que nous adoptons exige que l'innovation soit considérée autrement que comme la simple insertion mécanique de la nouveauté technique dans la production. Ce point de vue va au-delà de l'idée selon laquelle il existerait, en aval de l'invention, un usage social prêt ou disposé naturellement à intégrer une technique, un procédé ou un type particulier d'organisation des rapports sociaux. Nous soutenons qu'il faut plutôt considérer la présence continuelle du social tout au long du «processus de production de l'innovation », depuis l'intuition de l'inventeur jusqu'aux différents mécanismes permettant son institutionnalisation, en passant par les efforts nécessaires pour construire et diffuser l'usage social de l'invention.

Ce point de vue ne nie pas le rôle du marché dans la production de l'innovation, mais postule que celui-ci est enchâssé dans le social. L'analyse de ce lien nous apparaît essentielle pour comprendre les différentes dynamiques de développement qui prennent place sur des territoires, spécifiques certes, mais en articulation avec des processus globaux. Notre analyse de la place du social dans les processus d'innovation prend dans cet article la forme d'un survol, sommaire il va sans dire, des différentes perspectives élaborées pour étudier le processus de l'innovation. Nous proposons une démarche qui conduira à revisiter les différentes étapes qui ont jalonné l'évolution du concept d'innovation. 
Le texte est divisé en quatre parties. Dans un premier temps, nous rappelons certaines bases posées par les analyses fondatrices de Schumpeter et de Veblen, tout en constatant l'absence d'une réflexion spécifique sur l'innovation de la part des classiques de l'analyse sociale. Dans un deuxième temps, nous abordons l'innovation à partir des propositions des économistes évolutionnistes. Nous reconstruisons l'élaboration d'une explication qui a vu dans l'innovation sociale un processus cognitif qui se heurte à des résistances sociales localisées, ce qui pose le problème de la diffusion sociale et territoriale de l'innovation. Dans un troisième temps, nous mettons l'accent sur le renouvellement du concept d'innovation, renouvellement amorcé par des auteurs qui proposent un cadre conceptuel et méthodologique qui voit l'innovation comme un construit social aux multiples phases et aux multiples facettes. Puis, dans un quatrième temps, nous concluons en insistant sur le lien fort existant entre socialité, territorialité et marché.

Nous soutenons que l'innovation est une construction sociale et territoriale, dont la production et les effets dépendent des contextes socio-économiques conflictuels et hiérarchisés, aussi bien locaux que mondiaux. Dans cette optique, le territoire médiatise et institue des arrangements d'acteurs productifs, des organisations et des preneurs de décision, permettant ainsi l'émergence de cultures d'innovation spécifiques, mais pas isolées ni indépendantes de contextes plus globaux.

\section{Innovation et société : les analyses fondatrices}

Précisons-le dès le départ, dans les travaux des auteurs classiques de l'analyse de la société et de son évolution, la notion d'innovation ne reçoit pas une attention particulière. Cette absence d'intérêt s'explique, selon nous, par la conception que lesdits auteurs ont de la transformation des sociétés, une vision déterministe et unilinéaire. Cette conception, contre laquelle s'insurge Braudel (1985), habite la pensée d'auteurs aussi importants que Durkheim, Spencer et Marx. Ce n'est qu'à la fin du $19^{\mathrm{e}}$ siècle que le concept d'innovation fait une entrée déguisée dans le langage sociologique. Ceci se produit lorsque Tarde (1890) utilise la notion «d'imitation». Tarde explique l'évolution des sociétés par le cumul d'inventions au quotidien, « d'innovations », qui modifient graduellement le lot des comportements humains. Comme le propre de l'être humain, affirme Tarde, est d'imiter ses semblables, lorsqu'un nouveau comportement apparaît, ce dernier entraîne une réaction « épidémiologique » où « l'innovation » est imitée dès lors que les conditions le permettent. Cependant, Tarde se penche peu sur les conditions de cette imitation. Mais cette vision demeure marginale. Pour voir émerger une conceptualisation plus complète des processus innovateurs, il faut attendre, d'une part, les analyses de deux auteurs précurseurs, à savoir Schumpeter et Veblen, et d'autre part, que les anthropologues mènent à terme leur réflexion théorique sur l'évolution et la transformation culturelles à partir de grands travaux synthèses réalisés par Rivers, Boas, Malinowsky ou Kroeber ${ }^{2}$.

\footnotetext{
${ }^{2}$ Nous avons choisi ici de ne pas développer la piste des apports de l'anthropologie culturelle états-unienne et allemande.
} 


\subsection{L'entrepreneur innovateur de Schumpeter}

La principale contribution de Schumpeter à l'analyse de l'innovation est celle de l'entrepreneur innovateur. Schumpeter emprunte sa vision de l'entrepreneur ou du chef d'entreprise à la notion de Führershaft qui renvoie au fait que, dans tous les domaines de l'activité sociale, le chef a un rôle particulier. Les aptitudes de ce chef se résument essentiellement à l'initiative et à la volonté. C'est en la transposant dans le domaine de l'économique que Schumpeter en fait dériver la notion d'entreprise et d'entrepreneur. L'entreprise est l'acte de réaliser ; l'entrepreneur, l'agent qui réalise des combinaisons nouvelles de facteurs de la production (Tremblay, 1989).

Pour Schumpeter, l'entrepreneur n'est pas l'inventeur d'une découverte. Il est celui qui introduit cette découverte dans l'entreprise, dans l'industrie, dans l'économie, soit le responsable de sa diffusion à proprement parler. Selon lui, la société économique est dirigée par des décisions humaines, celles des entrepreneurs, et non pas par des idéologies ou des classes sociales abstraites. C'est en cela que la théorie de Schumpeter se distingue fondamentalement des perspectives déterministes et macrosociales évoquées ci-dessus. Ainsi, le changement découle de l'exercice réel d'une fonction et non de la fonction en tant que telle : c'est-à-dire que quelqu'un est « entrepreneur » lorsqu'il met en œuvre de nouvelles combinaisons ; il crée un contexte grâce auquel le cadre d'intervention du social s'élargit, se transforme. Seule cette action de dépassement correspond au rôle et à la fonction d'entrepreneur. Schumpeter campe son analyse dans le milieu économique et voit un acteur transformateur en toute personne en mesure de mettre en place une nouvelle combinaison d'arrangements dans le cadre d'une entreprise, d'une organisation à finalité économique.

Dans cette perspective, la fonction de l'entrepreneur consiste à surmonter une série d'obstacles. Selon Schumpeter, l'innovation est une réponse créatrice à ces obstacles. Trois grandes catégories de résistance à l'innovation peuvent être identifiées. Premièrement, l'entrepreneur innovateur agit dans un contexte d'incertitude, car compte tenu de l'information dont il dispose, il n'est pas assuré que son projet aboutira. Il peut avoir recours à des données rétrospectives, mais celles-ci apportent peu de certitude puisque personne ne les utilise dans la voie qu'il propose. La deuxième catégorie d'obstacles paraît relativement évidente. Schumpeter l'énonçait ainsi en 1935 : «Il est objectivement plus difficile de faire du nouveau que ce qui est accoutumé et éprouvé. » Enfin, la troisième catégorie est la plus importante à nos yeux. Il s'agit de la réaction du milieu social à l'égard de l'innovation, ou «à toute personne qui veut faire du nouveau» (Schumpeter, 1935). À cet égard, Schumpeter disait : « Il n'est pas suffisant de produire un savon satisfaisant, il faut encore entrấner le peuple à se laver ${ }^{3}$. » Cette métaphore est toujours d'actualité. Elle évoque le problème de la construction sociale de l'usage de l'invention, ce qui est le propre de l'innovation.

Si ses travaux ont permis d'isoler le rôle de l'entrepreneur comme agent central du changement dans les organisations à caractère économique, Schumpeter n'a pas été jusqu'à étendre le rôle d'entrepreneur à d'autres types d'acteurs du social, du politique, du culturel. Pour saisir toute la complexité liée à la reconnaissance de l'usage, il faut faire le lien entre les travaux de Schumpeter et ceux de Veblen.

\footnotetext{
${ }^{3}$ Il s'agit ici d'un extrait de Business Cycles (p. 243-244) repris dans Marty (1955 : 92).
} 


\subsection{Veblen et le rôle des technologies}

L'œuvre de Veblen constitue un apport important à l'analyse économique de l'innovation, mais surtout à la réflexion globale et interdisciplinaire sur ce sujet. Pour Veblen, tout comme pour Schumpeter, la technologie ou, selon ses mots, «l'état des techniques » (state of industrial arts), est le facteur économique primordial et déterminant de l'évolution sociale. Ce que Veblen entend par «technologie » se résume à deux séries d'éléments : d'une part, un système d'outils, d'instruments, de machines, et, d'autre part, ce qui constitue d'ailleurs pour nous l'aspect le plus important, un savoir-faire. Veblen utilise aussi les expressions « capital intangible», «capital collectif » et «avoir immatériel » en faisant référence à ce savoir-faire technique qu'il juge plus important que les outils et les instruments qui constituent le capital physique. Veblen voit la technologie comme une « possession indivise du groupe social, alors que les instruments que suscite cette technologie peuvent fort bien être appropriés privément » (Corbo, 1973).

Veblen considère que la technologie ne sera efficace que si elle trouve les conditions matérielles appropriées, si l'on dispose des forces matérielles requises, et si elle se trouve dans un milieu de diffusion ou un «milieu de propagation » approprié. Dans une certaine mesure, la théorie de Veblen est donc empreinte d'un certain « déterminisme technologique »; cependant, Veblen fait intervenir d'autres forces qui contribuent à atténuer ce déterminisme que l'on serait porté à relever. Parmi ces autres forces, mentionnons la «culture ». En effet, Veblen est d'avis que la technologie n'est pas une réalité statique, mais bien une réalité dynamique « qui évolue constamment et dont le degré d'efficacité dépend de certaines conditions précises ». « S'il est vrai que la technologie influence la culture, il est aussi vrai que la culture peut faciliter aussi bien qu'inhiber l'efficacité et le progrès de la technologie » (Corbo, 1973, p. 295). Ici encore, on se rapproche d'une vision globale et non pas seulement économique de la technologie.

En résumé, pour Schumpeter, l'innovation réside dans le processus qui mène à la généralisation, voire à la création de l'usage social de l'invention. Si l'entrepreneur s'occupe de la construction de l'usage, il le fait en créant lui-même quelque chose que l'inventeur n'avait pas, ce qui constitue aussi en soi une invention. À cet égard, l'analyse de Veblen complète et va même au-delà de l'analyse de Schumpeter lorsqu'il met en relief les effets de réciprocité entre la technique/technologie et l'environnement social. En effet, pour lui, non seulement les technologies ont-elles une incidence sur l'environnement culturel et institutionnel, mais cet environnement institutionnel exerce lui-même un effet sur les technologies. Il y aurait ainsi une forme de réciprocité d'effets entre le contexte social et les technologies (ou l'innovation, terme que nous préférons, mais que Veblen n'utilise presque pas).

\section{L'approche évolutionniste : de l'effet cyclique à l'effet spatial}

Comme nous venons de le voir, les travaux de Schumpeter et de Veblen constituent des antécédents importants pour l'élaboration d'une analyse qui enchâsse l'innovation dans le social. Mais ce sont les économistes dits «évolutionnistes » qui complètent leur œuvre en proposant une vision globale de l'innovation. Pour ce courant, l'innovation est un processus (Freeman et al., 1982), un « processus qui transmet des impulsions, en reçoit, raccorde les idées techniques nouvelles et les marchés » (Le Bas, 1995), un processus de résolution 
de problèmes, un processus d'apprentissage qui met en jeu des connaissances, des savoirs, des compétences, des savoir-faire, des capacités et des aptitudes (Winter, 1987).

\subsection{L'effet cyclique de l'innovation}

Selon les économistes évolutionnistes, le processus d'innovation est situé dans une organisation, une entreprise, ce qui les rapproche de la vision schumpeterienne (Dosi, 1988). Chez Schumpeter, il s'agissait dans un premier temps de petites ou de moyennes entreprises qui étaient le lieu premier de l'innovation, alors que la concentration du capital aurait au fil des ans donné lieu à la domination des grandes entreprises et de leurs départements de recherche et développement. Chez les évolutionnistes par contre, ces deux lieux (PME et grandes entreprises) ne se succèdent pas nécessairement dans le temps, mais peuvent au contraire coexister.

Cela nous semble mieux correspondre à la réalité actuelle où, selon les secteurs et selon le degré de maturité du secteur en question, ce sont tantôt les PME, tantôt les grandes entreprises qui dominent le processus d'innovation. D'ailleurs, ces deux régimes d'innovation peuvent fort bien s'expliquer par la phase dans laquelle se trouve une industrie donnée (Dosi, 1988). En phase d'émergence d'une industrie (on peut penser au multimédia, aux biotechnologies, à l'optronique, etc.), Dosi note que l'innovation tend à procéder par essais et erreurs ; les entrepreneurs prennent des risques, de nouvelles technologies apparaissent, ce qui donne lieu à la naissance de nouvelles entreprises. Au contraire, dans la phase de maturité (pensons aux secteurs de l'automobile, de l'acier, etc., où les marchés sont assez saturés), généralement caractérisée par une organisation de marché oligopolistique, les changements technologiques et l'innovation en général constituent une des armes de la concurrence, sinon la principale.

En effet, toute organisation tend à systématiser ses pratiques dans un cadre culturel organisationnel qui le rend réfractaire au changement. Cette situation est bien mise en lumière par la théorie dite «path-dependency » (North, 1990 ; Arthur, 1994 ; Mahoney, 2000). Selon ce cadre paradigmatique, les organisations sont confrontées à un certain conservatisme à l'égard de l'innovation en raison du poids des structures internes. Le recours à l'innovation se fait dans un contexte forcé où des pressions externes, en termes par exemple de perte de position dans le marché, interviennent pour forcer une rupture au sein de l'organisation. L'innovation apparaît dès lors comme une stratégie réactive et non comme une mesure pro-active.

Le potentiel dynamisant d'une innovation n'est donc pas éternel. Il s'épuise, ce qui explique les révolutions technologiques ainsi que l'apparition de nouveaux cycles technologiques et la désuétude des anciens. Comme l'a expliqué Vernon (1976), se rapprochant ainsi des analyses de Dosi (1988), ce potentiel est en relation étroite avec le cycle de vie d'un produit. Les travaux de Vernon montrent que le produit amorcé par l'innovation passe par une série de phases. Celui-ci distingue cinq phases : la nouveauté, la croissance, la maturité, la standardisation et le déclin.

L'effet dynamisant de l'innovation se fait sentir dans les premières phases, alors que la fabrication du nouveau produit crée de nouvelles occasions de marché, attire des capitaux et donc génère de nouvelles entreprises, y compris de petites entreprises, où l'on développe de nouveaux usages et des nouvelles variétés du produit. Par la suite, la création est remplacée par une production de masse aux normes plus rigides, plus concentrées, et le potentiel 
dynamisant de l'innovation diminue. Comme résultat de l'analyse du cas des États-Unis, Vernon propose une explication de l'effet de l'innovation sur le développement économique. Selon cette explication, le développement est lié à la capacité d'un pays, ou d'un milieu régional ou local, de se spécialiser dans la fabrication de produits situés dans leurs premières phases et de s'en dégager par la suite à mesure de l'avancement du cycle pour réorienter la spécialisation économique vers d'autres produits émergents. Il pose ainsi les jalons d'une nouvelle stratégie de développement axée sur la capacité d'innover, où la croissance est fonction de la spécialisation dans les premières phases de la production des biens.

\subsection{L'effet spatial de l'innovation}

Conformément à la perspective évolutionniste, les innovations ont un effet sur des dynamiques temporelles. Mais elles ont aussi un effet majeur sur le territoire. Cet effet spatial réside dans le fait que l'innovation relève de la combinaison de la découverte technique ou de l'invention et de la capacité organisationnelle et économique d'une collectivité de les mettre en valeur. Or, cette combinaison est un phénomène ponctuel. Comme l'affirme Perroux (1986), l'innovation n' apparaît pas partout ni en même temps. Elle apparaît dans des endroits précis où elle induit des changements dans les façons de produire et, partant, de consommer, où elle change les normes productives, et à partir d'où elle se diffuse.

Perroux situe la diffusion de l'innovation dans le contexte d'un processus conflictuel qui oppose, d'une part, les pratiques de production et de consommation induites par l'innovation, et, d'autre part, le potentiel d'un milieu pour s'y adapter. Mais s'y adapter ne signifie pas simplement imiter, comme l'a postulé Rostow (1960) dans son étude sur les étapes de la croissance économique. Cela fait référence aussi, et surtout, aux actions volontaires menées conjointement par des entrepreneurs et des organisations pour s'approprier le processus innovateur, le développer, le prolonger, et générer une capacité dynamique récurrente. Cela implique évidemment divers types d'innovation (Perrin, 1985).

Une question se pose alors : comment un milieu peut-il s'adapter de façon à créer des ensembles productifs capables de profiter de l'effet des innovations ? Poser ce problème suppose renverser le point d'observation. Il faut se demander comment les collectivités réagissent à la diffusion de l'innovation. Plutôt que de voir comment l'innovation agit, il faut alors voir comment on peut se reconvertir aux nouvelles pratiques productives induites par l'innovation, soit en se spécialisant dans les types de production qui ont un potentiel dynamisant, soit en mettant de l'avant des conditions sociales qui rendent la collectivité apte à innover (Fontan, Klein, Lévesque, 2003).

Selon l'analyse évolutionniste, l'innovation est donc vue comme un processus social qui se rattache aussi bien aux technologies ou systèmes techniques, qu'au marché des biens et au marché du travail (Le Bas, 1995), ce qui en fait un processus incertain, bien qu'il ne soit pas totalement aléatoire. L'entreprise intervient à l'interface de ces divers éléments. Elle effectue alors des médiations, des choix, dans le cadre de cet ensemble de faits sociaux dans lequel elle s'inscrit. Selon cette vision, la diffusion des nouvelles pratiques productives induites par l'innovation se réalise à travers les changements des normes productives, des changements communiqués d'une entreprise à l'autre, et donc d'un lieu à un autre, grâce à leurs interrelations productives. Cette diffusion se fait d'abord dans les limites des entreprises qui contribuent à la production induite par l'innovation, pour ensuite se généraliser à travers des filières qui comprennent des combinaisons d'entreprises et d'acteurs. 


\subsection{L'effet systémique territorialisé de l'innovation}

La diffusion du changement s'accompagne, voire provoque des changements de type social qui relèvent de la structure sociale et des caractéristiques culturelles des divers milieux. Ces changements concernent l'aptitude des collectivités à s'adapter au changement technologique induit par les différents types d'innovation et à se les approprier, générant ainsi à leur tour une capacité récurrente de mettre en valeur des innovations et de produire la croissance. La combinaison des changements sociaux, lesquels incluent la structure organisationnelle et sociale d'une collectivité, ainsi que la capacité de se mettre au diapason des innovations diffusées à travers les filières, constitue des conditions pour l'émergence de systèmes productifs caractérisés par l'innovation dans des régions et des milieux précis (Lundvall, 1988 ; Wolfe, 2002 ; Braczyk, Cooke, Heidenreich, 2003).

Plusieurs chercheurs ont abordé les rapports de l'innovation au territoire dans ce type de lieux en se servant des notions de «milieux innovateurs » (Aydalot, 1986 ; Maillat, 1992), de district industriel (Becattini, 1991 ; Piore et Sabel, 1984), ou de technopoles (Benko, 1991). Dans tous les cas, nonobstant les différences d'approche, ces notions ont été utilisées pour désigner des modalités d'agencement de la technologie, du territoire et des organisations d'une collectivité (Storper, 1997). Il en découle des collectivités où production et société s'imbriquent en configurant des systèmes territoriaux de production. La coordination des différentes phases de ces systèmes et le contrôle de la régularité de leur fonctionnement ne sont pas assujettis à des règles préétablies et à des mécanismes hiérarchiques, mais au contraire sont soumis à la fois au jeu automatique du marché et à un système de sanctions sociales infligées par la communauté. La contiguiité territoriale permet au système territorial des entreprises de tabler pratiquement sur des économies d'échelle liées à l'ensemble du processus productif, sans perdre toutefois de leur flexibilité et de leur adaptabilité aux divers aléas du marché grâce à la segmentation de ce processus (Klein, Tremblay, Fontan, 2003).

L'effet d'agglomération de ces systèmes territoriaux incite à la mise en place de coopérations locales, permettant de prendre en charge collectivement un ensemble de problèmes productifs individuels et d'assurer ainsi une gouvernance locale. C'est en cela que réside la synergie induite par ce type de systèmes. Cette synergie est rendue possible par le fait que les entreprises s'inscrivent dans des processus de production de nature équivalente et que l'objectif des processus d'apprentissages collectifs mis en place vise à résoudre les difficultés communes. La mise en œuvre de ces solutions implique des infrastructures nouvelles et s'exprime par la volonté de renforcer les partenariats entre les grandes firmes, les PME, les institutions de haut savoir (universités, instituts de recherche), le pouvoir local (municipalités, organismes locaux) et les institutions gouvernementales.

L'analyse des rapports de l'innovation au territoire met en relief l'effet de système créé par le renforcement des liens entre les acteurs économiques, sociaux, politiques et culturels partageant un même espace géographique, dans un contexte d'interrelations réticulaires construites à l'échelle mondiale. Le lieu est plus qu'une localisation. Il est un système. Il existe un « effet de lieu » qui oriente l'action des acteurs. Cet effet est économique, politique, social, culturel et idéologique. C'est l'effet de lieu qui conduit à la structuration hiérarchisée de systèmes locaux, à la structuration du local, en tant que résultat des agencements territoriaux des étapes et acteurs d'un réseau globalisé (De Bresson et Amesee, 1991 ; Holbrook et Wolfe, 2002 ; Tremblay et al., 2003). 


\section{Le renouvellement du concept d'innovation : vers l'innovation sociale}

La production récente sur le thème de «innovation et société » sort des sentiers tracés par les travaux des économistes évolutionnistes et par les auteurs qui s'en inspirent. Les écrits de Chambon, David et Deverey (1982) sur l'innovation sociale, de Flichy (1995) sur l'innovation technique ou ceux de Alter (2000) sur l'innovation ordinaire, font état de l'émergence d'une nouvelle approche de l'innovation. Pour cette nouvelle approche, il s'agit moins d'expliquer les liens entre l'innovation et la croissance économique (Amable, Barré et Boyer, 1997) que de comprendre les processus hétérogènes d'innovation porteurs de changement social aux échelles méso-sociale et micro-sociale.

Le renouvellement du concept d'innovation est aussi en corrélation avec la redécouverte des travaux de Polanyi (1944), qui redéfinit le champ de ce qui est économique. Rappelons que Polanyi définit l'économie comme un ensemble dynamique de processus sociaux en continuelle transformation dont découlent des formes d'intégration relevant de la réciprocité (logique symétrique), de la redistribution (logique centralisatrice) et de l'échange (logique de marché). Dans cet ensemble de processus, l'innovation peut être vue comme étant traversée par un double mouvement d'appropriation et de territorialisation.

Le mouvement d'appropriation est associé au processus de définition de l'usage social de l'invention, donc à sa propriété et aux normes entourant la généralisation de son usage. Ce processus d'appropriation se fait généralement dans la négociation, mais aussi dans l'imposition. Quant au mouvement de territorialisation, il consiste, selon nous, en la définition du cadre spatial de l'innovation, ce qui comprend l'imbrication des territoires dans une hiérarchie spatiale. Une hiérarchie spatiale spécifique au domaine visé par l'innovation se met en place, puisque l'usage social suppose une mise en relation différente d'éléments sociaux, c'est-à-dire des déplacements d'objets matériels et immatériels. Le mouvement de territorialisation structure à son tour les modalités de reproduction du social en agissant sur les flux existants : les renforçant, les réduisant, les orientant, les réorientant (Fontan, Klein, Lévesque, 2003). Il y a bien des liens entre innovation et territoire, comme nous l'avons rappelé ci-dessus, mais ces liens prennent place dans un contexte de redéfinition des hiérarchies sociales et des relations de pouvoir.

\subsection{L'expérimentation sociale et les relations avec le pouvoir}

La revue française Autrement a amorcé une réflexion au milieu des années 1970 sur l'innovation dans un contexte de relations de pouvoir. Cette réflexion a mis à contribution des penseurs pour lesquels l'innovation sociale correspond à de nouvelles expériences formelles ou informelles — à caractère social. L'innovation est analysée surtout sous l'angle politique en lui donnant une fonction particulière, celle d'être le moyen à partir duquel il est possible de transformer la société. C'est ainsi qu'est introduite dans la réflexion sur l'innovation la notion d' « innovation sociale », caractérisée selon Chambon, David et Deverey (1982) par trois aspects :

1) Le premier est d'ordre explicatif. L'innovation sociale n'est pas gratuite, elle est le produit d'un besoin, d'un désir, d'une aspiration, ou, encore, elle découle d'une recherche de solutions à un problème social. Fait important à noter, cette réponse se construit localement. Pour les différents auteurs concernés, le local est le lieu par excellence d'émergence de l'innovation sociale. Elle ne découle pas des mécanismes ou des processus nouveaux 
mis en scène par les grandes organisations ou institutions, mais des actions localisées et localisables. En ce sens, bien qu'une politique gouvernementale puisse difficilement être considérée comme une innovation sociale, elle peut être traitée comme étant l'appropriation par l'État d'une innovation mise de l'avant à l'échelle locale.

2) Le deuxième aspect est d'ordre moral et politique. L'innovation est politiquement orientée. Elle vise une amélioration de la qualité de vie. Elle apparaît en réponse à ce qui est vu comme l'incompétence des grandes institutions sociales considérées comme incapables d'assurer cette qualité. Le projet politique repose davantage sur le «faire autrement » que sur le modèle autoritaire et hiérarchisé des grandes institutions. L'innovation ainsi vue n'est pas nécessairement en rupture avec les grandes institutions, ce qui permet qu'elle puisse remonter la filière institutionnelle et provoquer des changements au sein de cette dernière. C'est ce que Morin présente comme « la déviance qui en arrive à faire tendance » (Autrement, 1976, p. 110).

3) Le troisième aspect est d'ordre économique : pour que les innovations perdurent, il leur faut un soutien financier. Plus les besoins financiers sont importants, plus il est difficile de ne pas faire financer l'expérience sociale par l'État. Se pose alors la question de la difficile relation entre l'innovation sociale et l'État.

Pour faire reconnaître l'usage social d'une expérience novatrice, l'analyse des tactiques utilisées par des innovateurs sociaux, selon Chambon, David et Deverey (1982), rend compte d'une stratégie de « court-circuitage », c'est-à-dire de contournement des obstacles, pour faciliter une mise en relation entre l'entrepreneur, au sens schumpeterien, et les décideurs de la sphère politique. Ce contournement fait en sorte que l'innovateur social va passer outre tout ce qui se présente comme obstacle entre lui et un lieu de pouvoir. L'innovateur individuel ou collectif cherche donc à réunir tous les moyens qui lui permettront d'influencer tout preneur de décision. La finalité de son action est d'établir une relation de confiance avec un décideur afin de réduire les zones d'inconfort et d'incertitude qui empêchent la reconnaissance de ses propositions ou de ses demandes par des décideurs politiques.

L'entrepreneur social entreprend donc un travail d'apprivoisement des réseaux sociaux d'influence. Sur ce point, qui est extrêmement important, ce n'est pas tant la nature particulière d'une stratégie utilisée par un acteur qui nous intéresse que la signification de cette dernière, à savoir l'idée de mobilisation de ressources pour contourner des obstacles, pour faire émerger une « décision » capable de modifier le cadre d'action dans la société (une nouvelle loi, de nouvelles normes). La construction de l'usage social, la cristallisation de l'innovation sociale se nourrissent de solutions mises en forme pour trouver une réponse effective au conflit qui oppose l'entrepreneur social à un milieu qui lui est hostile. L'enjeu tient à la diffusion et à la reconnaissance de la légitimité d'un «concept ou d'un projet social » : les entreprises d'insertion dès 1980, le commerce équitable au début des années 1990, les revendications alter-mondialistes depuis le milieu des années 1990.

On peut ici établir un parallèle avec les résistances sociales évoquées par Veblen. Pour surmonter les résistances, l'entrepreneur social utilise un réseau non pas construit au hasard, mais directement lié aux chaînes décisionnelles qui lui sont favorables. Et ce réseau se construit non pas parce que l'acteur visé, l'élu devenu ministre par exemple, est socialement enclin à la nouveauté, mais parce que ce dernier trouve un avantage politique à cette reconnaissance. Le politicien et l'innovateur jouent la carte du capital politique qu'ils cher- 
chent à valoriser par des alliances stratégiques. La médiation entre l'innovateur et le législateur ne se nourrit pas de générosité, mais elle s'alimente d'intérêts convergents et de compromis.

\subsection{La construction des réseaux sociaux d'innovation}

Dans la lignée de la sociologie interactionnaliste de Mead (1934), les travaux de Lazarsfeld et al., (1944) et de Katz (1955), pour ne mentionner que ces deux chercheurs, mettent en relief le fait que l'échange d'information ne se fait pas sans filtrage social. L'échange d'information entre un émetteur et un récepteur de message se réalise en fonction d'une reconnaissance préalable de l'utilité de l'information par un leader d'opinion, une personne influente et significative pour le récepteur du message. Le circuit de la communication comprend une part de doute, de méfiance, de peur face à la nouveauté, ou encore une part d'incommunicabilité de l'information. Le leader d'opinion est alors perçu comme un médiateur positif ou négatif qui permet de lever ou de confirmer le doute, ou encore d'interpréter l'information de façon telle que le message puisse ou ne puisse pas passer.

Ce constat de communication procédant par un flux à deux temps (two step flow) implique que la construction de l'usage social d'une invention a tout avantage à passer par l'intermédiaire de leaders d'opinion. L'entrepreneur social désirant voir son invention se diffuser se met en relation avec les leaders d'opinion concernés. Ce dernier point introduit l'apport des travaux de Latour (1988) et de Callon (1989) sur l'association étroite qu'il y a entre innovation et réticulation. Selon Callon, l'innovation n'est pas le fruit d'une intuition géniale issue du cerveau d'une seule personne. Au contraire, l'innovation est un processus auquel participe un nombre important d'acteurs, lesquels collaborent chacun à sa façon à la production de ce qui, après coup, paraît être un produit unique.

L'innovation s'élabore ainsi dans la transformation de l'intuition. Elle relève de l'ordre du processus et non de l'état de fait. Elle prend un peu plus de consistance au fil des contributions de chacun des acteurs interpellés par le processus innovateur. Ces contributions ne se font pas sans conflits. Il y a négociation entre des points de vue plus ou moins convergents et parfois divergents : l'innovation s'établit ainsi dans la négociation d'une entente, d'une convention. L'innovation technique, nous dit Callon, est une information qui se fabrique au fur et à mesure que se réalisent des négociations entre des acteurs rattachés à des réseaux au sein de systèmes hiérarchisés d'innovation.

\section{L'innovation enchâssée dans le local et le global, dans l'individuel et le collectif}

Étudier l'innovation, c'est rendre compte d'un processus porteur d'un ensemble de dimensions appelées à être déterminantes et à occulter les autres en fonction de la nature de ce qui est innové. Le processus d'innovation est certes technique et économique, il est tout autant social que politique et évidemment il a une portée culturelle. Étudier une innovation c'est mettre en lumière les particularités sociales, techniques, économiques, politiques et culturelles qui sont mises de l'avant par des acteurs individuels et collectifs. Cet ensemble d'éléments trouve corps et sens du fait qu'il prend place à une période et en un lieu précis. Dès lors, tant le territoire que la dimension temporelle sont importants et donc à prendre en considération. 
Notre hypothèse veut que l'innovation soit conditionnée par un contexte social. Ainsi, pour nous, la dynamique de l'innovation sociale met en scène des acteurs occupant des positions au sein d'un ensemble d'arrangements institutionnels. Puisque ces ensembles, comme le démontrent Hollingsworth et Boyer (1996), sont structurés géographiquement à partir de modalités différentes mais complémentaires de régulation des systèmes sociaux, l'innovation sociale opère en fonction de ces niveaux de régulation. On est alors en mesure de parler de l'existence d'un ensemble de systèmes d'innovation sociale appartenant à des espaces géographiques allant de l'espace mondial à l'espace local en passant par les espaces intermédiaires (continental, national et régional). Nous sommes ainsi en présence d'une carte spatialisée articulant une combinaison de systèmes d'innovation, dont certains sont d'ailleurs transfrontaliers (Klein, 1998). Si l'innovation n'échappe pas au déterminisme culturel du monde et du système social qui l'enchâsse, ce déterminisme culturel est lui-même hiérarchisé autour de la réalité de l'économie-monde et prend place au sein d'un processus de mise en relation sociale hautement actionnaliste ou interactionnaliste. Cette réalité sociale, fort bien étudiée et décrite par Alter (2003), permet de comprendre comment les acteurs entrent en interactions fortes ou faibles en fonction d'enjeux et de processus visant l'appropriation ou la non-appropriation de ressources et de pouvoirs, favorisant par le fait même l'intégration ou la déviance, la domination et la dominance.

Notre vision de l'innovation insiste sur la construction sociale de l'innovation et sur les processus et les interrelations sociales et territoriales qui interviennent à tous les niveaux. Nous avons surtout voulu proposer une perspective globale qui voit les acteurs, ainsi que les territoires, comme des éléments en interrelation, c'est-à-dire en tension et en convergence. Dans cette perspective, l'innovation ne porte pas en soi une fonction positive liée au progrès social. Le progrès, en tant que changement subjectif dans la configuration des rapports sociaux, découle des choix effectués par les acteurs lors du double mouvement d'appropriation et de localisation d'une invention. L'usage social devient positif ou négatif pour une communauté en fonction de ce qu'en feront les acteurs sociaux. L'innovation sociale nous apparait donc comme un processus multiforme et multidimensionnel de production et de rénovation de l'existant, dans le but de produire du changement social, et ce, à diverses échelles.

L'action socialement innovante représente une médiation qui permet d'apporter une réponse à un besoin ou à un désir formulé par des acteurs sociaux pour trouver une solution à un problème social. L'action socialement innovante relève du domaine de la stratégie puisqu'elle agit sur le système d'action d'une organisation ou d'une communauté. L'action socialement innovante signifie une plus grande structuration du social 1) en amont de l'innovation, par la redéfinition des orientations culturelles, 2) lors de sa mise en œuvre, par les nouvelles modalités de gestion des rapports sociaux, la concertation et le partenariat par exemple, et 3) en aval de celle-ci, par les pressions exercées pour changer les habitudes individuelles et collectives de consommation. C'est ainsi qu'il se construit des milieux où l'invention et la nouveauté émergent plus facilement et où le cycle conduisant à la reconnaissance de son usage social et à son institutionnalisation s'accélère, ce qui met en place des systèmes d'innovation.

Ce rapide survol des différentes façons d'aborder la place de la société dans les processus innovateurs nous permet de conclure que l'innovation découle d'actes certes individuels, au sens d'un leadership exercé par un individu, mais réticulés, encadrés, rendus possibles par un contexte social et culturel favorable ou défavorable, par un contexte 
économique plus ou moins conciliant et par des négociations prenant place entre des acteurs plus ou moins en accord avec la définition d'un nouvel usage social. Donc, l'innovation sociale englobe à la fois le fait d'innover, c'est-à-dire une action inventive orientée culturellement, produit de l'imaginaire ou du hasard, et le processus institutionnel de reconnaissance sociale de son usage, de son passage à l'institué.

\section{Références}

Alter, N., 2003. «Innovation organisationnelle, entre croyance et raison». In: Mustar, P., Penan, H. (Eds.), Encyclopédie de l'innovation. Paris, Economica, 71-88.

Alter, N., 2000. L'innovation ordinaire. Presses universitaires de France, Paris.

Amable B., Barré R., Boyer, R., 1997. Les systèmes d'innovation à l'ère de la globalisation. Paris, Economica.

Arthur, W.B., 1994. Increasing returns and Path Dependency in the Economy. Ann Arbor, University of Michigan Press.

Aydalot, Ph., 1986. «L'aptitude des milieux locaux à promouvoir l'innovation ». In : Federwish, J., Zoller, H. (Eds.), Technologies nouvelles et ruptures régionales. Paris, Economica, 41-58

Becattini, G., 1991. «Italian Districts: Problems and Perspectives ». International Studies of Management \& Organization 21(1), 83-90.

Benko, G., 1991. La géographie des technopôles. Paris, Masson.

Benko, G., Lipietz, A., (Eds.), 2000. La richesse des régions. Paris, Presses universitaires de France.

Braczyk, H.-J., Cooke, P., Heidenreich, M. (Eds.), 2003. Regional Innovation Systems. Londres, Routledge.

Braudel, F., 1985. La dynamique du capitalisme. Paris, Champs Flammarion.

Callon, M., 1989. La science et ses réseaux. Paris, La Découverte.

Chambon, J.L., David, A., Deverey, J.M., 1982. Les innovations sociales. Paris, PUF (Que sais-je ? 2014).

Corbo, C., 1973. Les théories épistémologiques et sociales de T.B. Veblen (1857-1929). Thèse de doctorat, Université de Montréal.

De Bresson, C., Amesse, F., 1991. « Networks of innovators ». Research Policy 20, 363-379.

Dosi, G., 1988. «The nature of the innovative process ». In: Dosi, G., Freeman, C., Silverberg, G., Soete, L., (Eds.), Technical change and economic theory. Pinter, New York, 221-238.

Flichy, P., 1995. L'innovation technique. Paris, Éditions La Découverte.

Fontan, J.-M., Klein, J.-L., Lévesque, B., 2003 (Eds). Reconversion économique et développement territorial : le rôle de la société civile. Québec, Presses de l'Université du Québec.

Freeman, C., Clark, J., Soete, L., 1982. Unemployment \& Technical Innovation: A Study of Long Waves \& Economic Development. London, Pinter.

Holbrook, J.-A., Wolfe, D. (Eds), 2002. Knowledge, Clusters and Regional Innovation. Montréal \& Kingston McGill-Queen's University Press.

Hollingsworth, G.R., Boyer, R., 1996. Contemporary Capitalism, the Embeddedness of Institutions. Cambridge, Cambridge University Press.

Katz, E., Lazarsfeld, P. F. (Eds), 1955. Personal Influence: The Part Played by People in the Flow of Mass Communications. Glencoe, IL, Free Press.

Klein, J.-L., 1998. « Mondialisation et État-nation : la restructuration territoriale du système-monde ». In : Klein, J.-L., Laurin, S. (Eds.), L'Éducation géographique. Conscience territoriale et formation du citoyen. SainteFoy, Presses de l'Université du Québec, 34-70 (2 édition : 1999).

Klein, J.-L., Tremblay, D.-G., Fontan, J-M., 2003. « Systèmes locaux et réseaux productifs dans la reconversion économique : le cas de Montréal ». Géographie, Économie, Société 5 (1), 59-75.

Latour, B., 1988. La vie en laboratoire. La Découverte, Paris.

Lazarfeld, P.F., Berelson, B., Gaudet, H., 1944. The people's choice. Duell, New York, Sloan \& Pearce.

Le Bas, C., 1995. Économie de l'innovation. Paris, Presses universitaires de France.

Levesque, Bourque, Forgues, 2001. La nouvelle sociologie économique. Paris, Desclée de Brouwer.

Lundvall, B.-A., 1988. «Innovation as an interactive process; from user-producer interaction to the national system of innovation ». In: Dosi, G., Freeman, C., Nelson, R., Silverberg, G., Soete, L. (Eds), Technical Change and Economic Theory. New York, Pinter, 349-369. 
Mahoney, J., 2000. « Path Dependency in historical sociology ». Theory and Society 29, 507-548.

Maillat, D., 1992. «Milieux et dynamique territoriale de l'innovation». Revue canadienne des sciences régionales 2 ; XV(2), 199-218.

Maillat, D., Quevit, M., Senn, L. (Eds) 1993. Réseaux d'innovation et milieux innovateurs. Neuchâtel, GREMI, EDES.

Marty, A.G., 1955. Analyse critique de l'œuvre de Joseph Schumpeter : (Eds.) Montana. Bruxelles, 250 p.

Mead, M., 1935. Sex and Temperament in Three Primitive Societies. New York, Morrow.

Morin, E., 1976. «Rencontres avec Paul-Henri Chombart, de Lauwe, Félix Guattari, Edgard Morin, Serge Moscovici ». Autrement 5, été, 98-116.

North, D., 1990. Institution, Institutional Change and Economic Performance. Cambridge, Cambridge University Press.

Perrin, J.-C., 1985. « Redéploiement industriel et aménagement du territoire: le cas français ». In : Boisvert, M., Hamel, P. (Eds), Redéploiement industriel et planification régionale, Montréal, Faculté de l'aménagement. Université de Montréal, 69-92.

Perroux, F., 1986. « Note sur la notion de pôle de croissance ». In : Savoie, D., Raynauld, A., (Eds.) Essais sur le développement régional. Presses de 1'Université de Montréal, Montréal, 27-37. (Version originale publiée en 1955.)

Piore, M., Sabel, C.F., 1984. The Second Industrial Divide. New York, Basic Books.

Polanyi, K., 1944. The Great Transformation. New York, Holt Rinehart.

Schumpeter, J. A., 1935. Théorie de l'évolution économique. Paris, Dalloz.

Storper, M., 1995. «La géographie des conventions : proximité territoriale, interdépendances hors marché et développement économique ». In : Rallet, A. et Torre, A. (Eds.), Économie industrielle et économie spatiale. Paris, Economica.

Storper, M., 1997. «Les nouveaux dynamismes régionaux : conventions et systèmes d'acteurs ». In : Coté, S., Klein, J.-L., Proulx, M.-U. (Eds.), Action collective et décentralisation. GRIDEQ, Rimouski, 1-17.

Tarde, G., 1890. Les lois de l'imitation. Paris, Félix Alcan.

Tremblay, D.-G., 1989. La dynamique économique des processus d'innovation. Thèse de doctorat en économie, Université de Paris 1.

Tremblay, D.-G., Klein, J.-L., Fontan, J.-M. et Rousseau, S., 2003. « Proximité territoriale et innovation. Une enquête sur la région de Montréal ». Revue d'Économie Régionale et Urbaine 5, 835-852

Veblen, T.,1899. The Theory of the Leisure Class: An Economic Study of Institutions. New York, The Macmillan Company.

Vernon, R., 1974. Les conséquences économiques et politiques des entreprises multinationales. Paris, Robert Laffont.

Wolfe, D., 2002. «Social Capital and Clusters Development in Learning Regions ». In: Holbrook, A. et Wolfe, D. (Eds.), Knowledge, Clusters and Regional Innovation. McGill-Queens University Press, TorontoMontréal, 11-38. 